\title{
c-myc null cells misregulate cad and gadd45 but not other proposed c-Myc targets
}

\section{Andrew Bush, Maria Mateyak, ${ }^{1}$ Kerri Dugan, Alvaro Obaya, ${ }^{1}$ Susumu Adachi, ${ }^{1}$ John Sedivy, ${ }^{1}$ and Michael Cole ${ }^{2}$}

\begin{abstract}
Department of Molecular Biology, Princeton University, Princeton, New Jersey 08544 USA; ${ }^{1}$ Department of Molecular Biology, Cell Biology, and Biochemistry, Brown University, Providence, Rhode Island 02912 USA
\end{abstract}

We report here that the expression of virtually all proposed c-Myc target genes is unchanged in cells containing a homozygous null deletion of c-myc. Two noteworthy exceptions are the gene cad, which has reduced log phase expression and serum induction in c-myc null cells, and the growth arrest gene gadd45, which is derepressed by c-myc knockout. Thus, cad and gadd45 are the only proposed targets of $\mathrm{c}-\mathrm{Myc}$ that may contribute to the dramatic slow growth phenotype of c-myc null cells. Our results demonstrate that a loss-of-function approach is critical for the evaluation of potential c-Myc target genes.

Received September 28, 1998; revised version accepted November 3, 1998.

Mutations that disrupt the regulation or expression level of the c-myc gene are frequently found in human and animal cancers (for review, see Henriksson and Lüscher 1996). In addition to having a role in uncontrolled cell growth, many observations indicate that $\mathrm{c}-\mathrm{myc}$ also regulates normal cell proliferation. First, c-myc is expressed in all dividing cells, and, conversely, expression is suppressed once cells withdraw from the cell cycle or terminally differentiate (Marcu et al. 1992). Second, antisense-mediated reduction of c-Myc suggests that it is required for efficient progression through the cell cycle (Yokoyama and Imamoto 1987). Third, ectopic expression of c-Myc or activation of a c-Myc-estrogen receptor fusion protein (Myc-ER) can in some circumstances induce quiescent cells to enter S phase (Armelin et al. 1984; Eilers et al. 1989). Despite substantial effort, the molecular mechanisms by which c-myc controls proliferation and tumorigenesis are not understood.

The c-Myc protein dimerizes with Max and recognizes the c-Myc/Max E-box consensus site (CACGTG) (Henriksson and Lüscher 1996). Because transfection of c-myc can transactivate promoters containing this binding site (Kretzner et al. 1992), it is generally believed that c-Myc functions by activating the expression of specific target

[Key Words: c-myc; transcription; target genes; cell cycle; proliferation]
${ }^{2}$ Corresponding author. E-MAIL mcole@molbio.princeton.edu; FAX (609) 258-2759. genes. However, the biggest impediment to understanding c-Myc function is the lack of a comprehensive set of c-Myc target genes. Numerous studies have attempted to identify c-myc targets, mainly using subtractive hybridization, differential display, or educated guessing. The list of candidate transactivation targets includes ornithine decarboxylase $(O D C), \alpha$-prothymosin, lactate dehydrogenase- $A$ (LDH-A), p53, ECA39, eIF4E, cad, $M r D b, r c l, R C C 1$, and cdc25A (Eilers et al. 1991; Benvenisty et al. 1992; Bello-Fernandez et al. 1993; Reisman et al. 1993; Wagner et al. 1993; Miltenberger et al. 1995; Galaktionov et al. 1996; Grandori et al. 1996; Jones et al. 1996; Lewis et al. 1997; Shim et al. 1997; Tsuneoka et al. 1997). All of the data supporting these genes as bona fide c-Myc targets come from transient or overexpression experiments; consequently, it is not clear whether any of the proposed target genes are necessary for the ability of c-Myc to control cell proliferation.

Although c-Myc clearly is instrumental in directing proliferation, recent studies indicate that it is not absolutely essential for cell growth. Cell lines were derived by targeted homologous recombination in a nontransformed Rat1 fibroblast line to knock out all c-myc expression, and these lines have no detectable N-myc or L-myc expression that might compensate for the absence of c-myc (Mateyak et al. 1997). Although the c-myc null cells continue to grow, they have a dramatically extended cell-cycle time that is nearly three times longer than their wild-type parent. The c-myc null cells have a prolonged $\mathrm{G}_{1}$ and $\mathrm{G}_{2}$ phase, arguing that c-myc functions in both of these phases of the cell cycle (Mateyak et al. 1997). Interestingly, S phase is not prolonged, suggesting that DNA replication is unaltered by c-myc knockout. These c-myc null cell lines provide a powerful new tool for the evaluation of potential c-Myc target genes. In this report we present an analysis of the proposed c-Myc target genes using this novel loss-of-function approach. The results suggest a significant re-evaluation of the proposed collection of c-Myc target genes and indicate that the transactivation of most known cellular targets is not linked to the ability of c-Myc to control the cell cycle.

\section{Results}

Stable ectopic expression of c-Myc does not hyperactivate the expression of proposed target genes in log-phase cells. One possible explanation for this is that the level of c-Myc in a normal cycling cell is saturating, and hence a target promoter is already maximally activated. The c-myc null cell lines provide an opportunity to directly test whether or not c-Myc is required for the basal expression of proposed target genes. The content of RNA and protein per cell is the same for c-myc wild-type and null cells in log phase, but we considered both the total cell number and total RNA quantitation in assessing specific mRNA levels.

We assayed mRNA levels in c-myc null cells and the wild-type parental line during log-phase growth using 
Northern blots that were quantitated with a PhosphorImager. Besides loading equal amounts of RNA per lane, the signals were normalized to the signal for GAPDH, which was previously found to have an equivalent level on a per cell and per RNA basis in a comparison of c-myc wild-type and c-myc null cells (Mateyak et al. 1997). The mRNA levels were quantitated for the genes $c d c 25 A$, ODC, a-prothymosin, MrDb, cad, eIF4E, LDH-A, RCC1, p53, and ECA39. With the exception of cad discussed below, the expression of all potential c-Myc target genes is unchanged by deleting c-myc (Fig. 1A). Because c-Myc is expressed continuously throughout the cell cycle in dividing cells (Hann et al. 1985), the data demonstrate that the level of these mRNAs is not dependent on c$\mathrm{Myc}$, and hence their expression must be under the control of other transcription factors. $c d c 25 \mathrm{~A}$ stands out as a potentially crucial putative c-Myc target gene, as an increase in its activity would be expected to stimulate progression through the $G_{1}$ phase of the cell cycle (Galaktionov et al. 1995). However, repeated attempts failed to show any change in the expression of $c d c 25 \mathrm{~A}$ in the absence of c-myc. These results have been confirmed in two independently derived c-myc null lines and are therefore not a result of clonal variation (data not shown). We conclude that the growth defect in the c-myc null cells cannot be attributed to a change in the expression of most proposed c-Myc transactivation targets.

In contrast, the expression of cad is reproducibly reduced about threefold in c-myc null cells when compared to the wild-type line (Fig. 1A). To determine if the defect in cad expression is due to loss of c-Myc, we reintroduced c-myc into c-myc null cells using retroviral transduction. Reconstitution of $\mathrm{c}-m y c$ re-established normal cad expression (Fig. 1B). Thus, cad appears to be uniquely dependent on c-Myc for normal expression in log-phase cells. cad gene expression is still readily detectable in the c-myc null line, indicating that other fac-

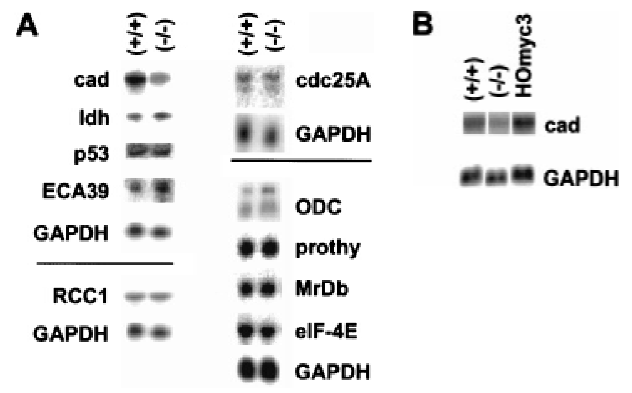

Figure 1. The expression of most proposed c-myc target genes is unchanged in c-myc null fibroblasts. $(A)$ Total RNA was isolated from exponentially growing parental c-myc wild-type $(+/+)$ or null $(-/-)$ cells and analyzed by Northern blotting. To ensure vigorous cell growth, RNA was isolated from subconfluent dishes 22-28 hr after replating. mRNA levels were measured three to five times for each gene with equivalent results for independent samples. The corresponding GAPDH expression level is shown for each panel of RNAs. (B) Total RNA was analyzed for c-myc wild-type TGR $(+/+)$, c-myc null HO15.19 $(-/-)$, and c-myc null cells reconstituted with a retrovirally transduced mouse c-myc cDNA, HOmyc3. tors contribute to expression during log phase growth. These factors may include SP1, which has binding sites that contribute to the basal activity of a reporter construct (Kollmar et al. 1994).

$\mathrm{c}-\mathrm{myc}$ is an immediate early response gene induced by mitogenic signals. It was possible that c-Myc might regulate target gene expression during the initial phase of growth factor stimulation, and this would be missed by examining expression in asynchronously growing cells. Therefore, we assayed target gene expression after treatment of serum-starved cells with $10 \%$ serum. In wildtype cells, the cad gene is strongly induced, with the mRNA reaching a peak at $\sim 12 \mathrm{hr}$ after mitogenic stimulation (Fig. 2A). This induction is virtually abolished in c-myc null cells, leading to an 11-fold difference between c-myc null and wild-type cells (Fig. 2B). The peak of cad induction takes place near the $G_{1} \rightarrow S$ transition in the parental line, which is at $\sim 13 \mathrm{hr}$ after mitogen stimulation. c-myc null cells enter S phase more slowly at 23-26 hr (Mateyak et al. 1997). Thus, the time course in Figure 2 covers the $G_{1} \rightarrow S$ transition in c-myc null cells, which corresponds to the peak of cad expression in the parental line. Extending the time of serum stimulation to $36 \mathrm{hr}$ gives no further induction of cad (data not shown); hence, cad induction is severely diminished and not just delayed.

The response of the other proposed c-Myc target genes to the c-myc knockout is variable (Fig. 2). For example, eIF4E shows virtually no difference between wild-type and c-myc null cells. On the other hand, the induction of $O D C, M r D b$, and $c d c 25 A$ mRNAs is somewhat reduced in c-myc null cells at early times after serum stimulation, although, after $24 \mathrm{hr}$ all of these genes are expressed at nearly equivalent levels with or without c-myc (Fig. 2). However, the physiological significance of the early kinetic differences is questionable when the Northern blots are quantitated in reference to a control mRNA, GAPDH (Fig. 2A,B). In both serum-starved and serumstimulated cells, there was a reduced level of GAPDH in c-myc null cells, which paralleled that found with most proposed c-Myc target genes. The level of L32 mRNA was reduced in c-myc null cells to an extent similar to $G A P D H$, indicating that other genes linked to general cell growth and metabolism are also affected (data not shown). Because there is no evidence that GAPDH or L32 is regulated by c-Myc, it is likely that these effects are indirect and may be due to the slower emergence of c-myc null cells from quiescence. We therefore normalized the expression of the proposed c-Myc target genes to GAPDH by dividing the ratio of the target gene signal in wild-type versus c-myc null cells by the ratio of GAPDH in wild-type versus c-myc null cells. When the data are examined in this way (Fig. $2 \mathrm{C}$ ), there is only a 1.5 - to 2-fold greater induction of some proposed targets $(c d c 25 A, O D C, M r D b)$ in the wild-type parental line at early times after serum stimulation, but even this small effect disappears by $24 \mathrm{hr}$. Thus, cad is the only gene whose activation is substantially dependent on c-Myc during mitogen stimulation, consistent with the Myc dependence observed in log-phase cells. 
A

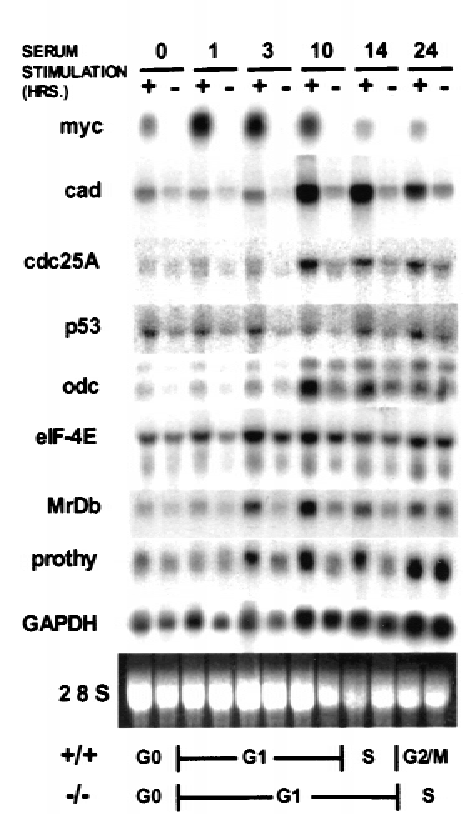

B
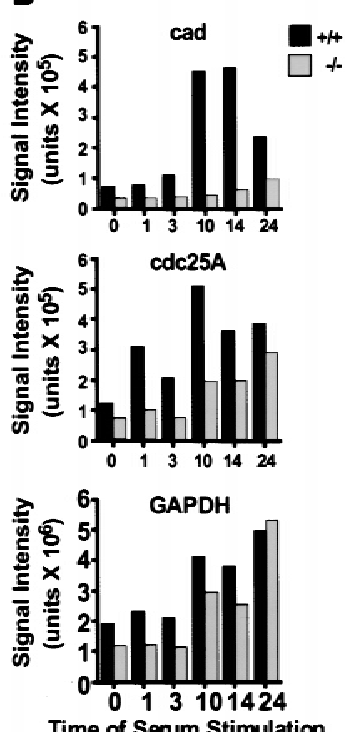

c

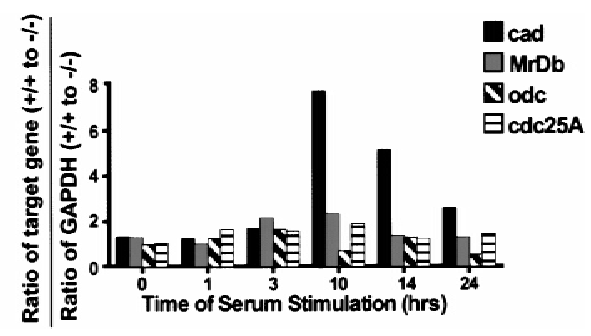

Figure 2. cad expression is greatly diminished by c-myc knockout during serum stimulation. c-myc wild-type and null cells were rendered quiescent by culturing in $0.1 \%$ serum for 48 $\mathrm{hr}$ and were then induced to re-enter the cell cycle with $10 \%$ serum medium. Total RNA was isolated at the indicated time points. The 14- and 24-hr RNA samples were slightly underloaded compared to earlier time points based on $28 \mathrm{~S}$ rRNA staining; hence, the relative hybridization signals for all genes are slightly under-represented at these points. $(A)$ Northern blots using the indicated gene probes. Cell-cycle transitions for the two cell types (bottom lines) are based on Mateyak et al. (1997), as described in the text. (B) Quantitation of bands in $A$. (C) Normalization of target gene signals using the ratio of GAPDH in c-myc wild-type to null cells.

As an independent assessment of mRNA expression to supplement the Northern blot analysis, the expression of GAPDH, rRNA, cdc25A, and p53 was examined by RNase protection during serum stimulation (Fig. 3). In this case, the data were normalized to equal cell number. As reported, c-myc null cells exhibit a reduced induction of rRNA and GAPDH mRNA after serum stimulation (Mateyak et al. 1997). The induction profiles of $c d c 25 A$ and $p 53$ were reduced to the same extent as GAPDH (Fig. $3)$, indicating that any difference between wild-type and c-myc null cells was likely due to an indirect reduction in cell-cycle progression in the latter that affects nontarget genes and proposed target genes equally.

The cell-cycle defect in c-myc null cells can be rescued

by reconstituting c-Myc expression through retroviral transduction (Mateyak et al. 1997), and this provides an assay for functional domains in c-Myc that are required for efficient cell-cycle progression. We were particularly interested in the c-Myc amino terminus, which has transactivation activity in transient assays. Furthermore, all of the biological activities of c-Myc are dependent on an evolutionarily conserved region in the amino terminus called Myc homology box II (MbII). To determine whether MbII is necessary for normal growth rates, we reconstituted the c-myc null cells with a c-myc $(\Delta \mathrm{MbII})$ mutant (Brough et al. 1995). The growth rate of the latter cells was still severely impaired (Fig. 4). This indicates that the MbII domain is essential for growth-promoting activity in nontransformed cells. However, it should be noted that the c-Myc $(\Delta \mathrm{MbII}) \mathrm{mu}-$ tant is not completely devoid of proliferative activity because the growth rate of the cells expressing this protein is faster than cells with no c-Myc, and this protein has been shown previously to have some residual activity in transformation assays (Brough et al. 1995).

In addition to its ability to transactivate, c-Myc can also repress cellular genes. The mechanism of c-Mycmediated repression remains unclear. However, functional domain mapping indicates that repression by cMyc is genetically inseparable from oncogenic transformation (Penn et al. 1990), in contrast to transactivation, which can be uncoupled (Bello-Fernandez et al. 1993;
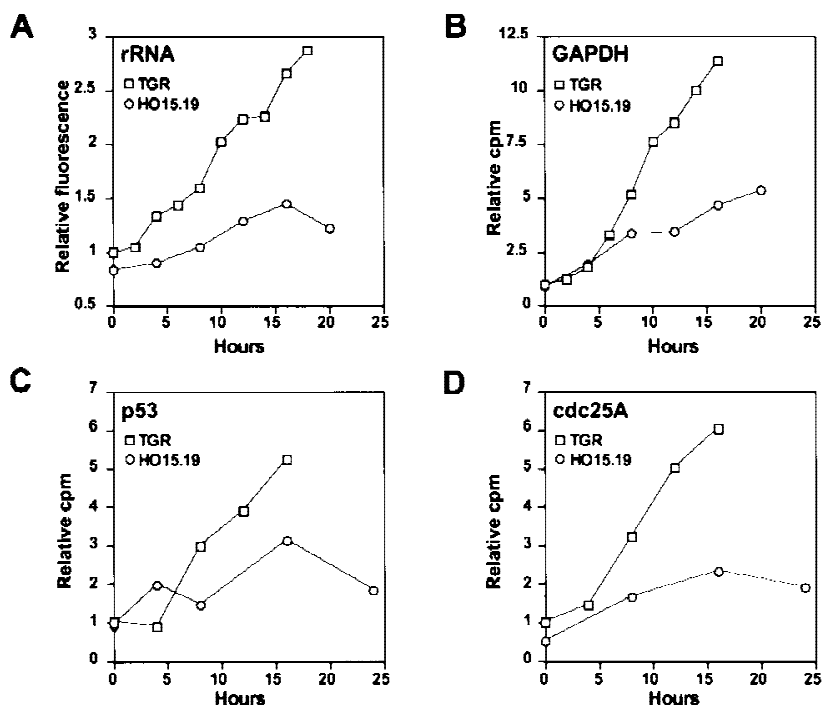

Figure 3. Serum regulation of rRNA, GAPDH, p53, and $c d c 25 A$ expression in c-myc null and wild-type cells. Quiescent cells were stimulated with serum, and RNA samples were harvested at the indicated times. The zero time point corresponds to the addition of serum. All values are expressed relative to the TGR-1 sample at $t=0$, which has been assigned a value of 1.0. (A) rRNA. Total RNA was resolved by agarose gel electrophoresis, and the ethidium bromide fluorescence in the 28S rRNA band was digitized. (B) GAPDH. Total RNA was analyzed by Northern blotting. $(C)$ p53. $(D) c d c 25 A$. Total RNA was analyzed by ribonuclease protection. The values were normalized to GAPDH internal controls, and the data are presented on the basis of equal cell number. 
Bush et al.

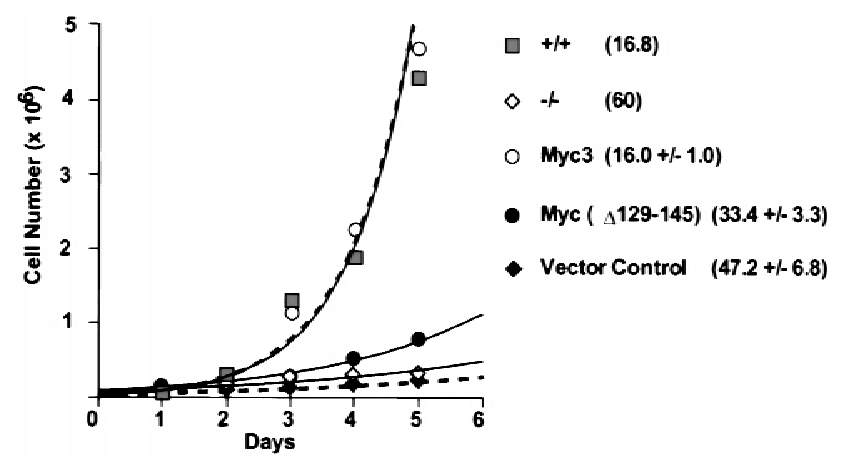

Figure 4. MbII is necessary for wild-type growth rate. c-myc (wild type) or c-myc with a deletion in MbII ( $\Delta 129-145)$ was reintroduced into c-myc null cells using the LXSH retrovirus, and clonal cell lines were analyzed for growth rate. Growth rates were determined by plating cells at low density $\left(1 \times 10^{5}\right)$ $10-\mathrm{cm}$ plate) and counting cells at the indicated time (in duplicate). The curves in $A$ depict the data from one representative experiment, and doubling times (mean \pm S.E.M.) were calculated using an exponential curve fit. Doubling time (hr) in parentheses.

Brough et al. 1995). It was therefore of interest to determine if target genes associated with c-Myc repression were misregulated in c-myc null cells.

The most thoroughly documented c-Myc-repressed gene is c-myc itself, a phenomenon often called autosuppression (Facchini et al. 1997 and references therein). During construction of the c-myc null cells, neo replaced exon 2 of c-myc by homologous recombination. Thus, neo expression should be controlled by the cis-acting sequences of the c-myc locus. Northern blots of RNA isolated from log-phase cells showed a clear neo-containing mRNA (Fig. 5A). Of more interest was the response of the endogenous c-myc promoter to ectopic c-myc expression. Reconstitution of wild-type c-myc expression led to repression of neo expression, indicating that the endogenous c-myc promoter is autosuppressed. This suppression is directly linked to the growth-promoting activity of c-Myc, because cell lines reconstituted with the defective c-Myc $(\Delta \mathrm{MbII})$ protein show no suppression of the endogenous c-myc promoter (Fig. 5A). Thus, the cmyc autosuppression pathway remains active in $\mathrm{c}-m y c$ null cells even though the expression of most candidate c-Myc target genes is unchanged.

Another cellular gene that is subject to Myc-dependent repression is gadd45 (Marhin et al. 1997). To determine whether c-myc is necessary for the repression of gadd45 mRNA levels, we performed Northern blots and RNase protection assays. In log-phase cells, gadd45 mRNA is significantly elevated (sixfold) in c-myc null cells compared to the wild-type parental line (Fig. 5B). Furthermore, reconstitution of wild-type c-Myc into the c-myc null line suppresses gadd45 levels to that found in the wild-type line (Fig. 5B). Similarly, the levels of gadd45 mRNA are higher in c-Myc-deficient cells during serum stimulation of quiescent cells (Fig. 5C). This difference extends to the protein level (data not shown), suggesting that the higher levels of gadd45 mRNA produce higher levels of gadd45 protein.

\section{Discussion}

The loss-of-function analysis in this study suggests a major re-evaluation of the contribution of c-Myc to the transcriptional regulation of proposed targets. Prior work has examined c-Myc target genes primarily through transient and overexpression approaches, and the data suggested that c-Myc could transactivate several target promoters from 3- to 10-fold (Henriksson and Lüscher 1996). However, to study the effects of ectopic c-Myc, it has been necessary to reduce endogenous c-Myc levels using growth factor deprivation. This growth factor deprivation influences many other aspects of cell physiology, including transcriptional control. Moreover, ectopic cMyc stimulates progression toward S phase under conditions in which cells would normally growth arrest, making it difficult to separate a direct effect of c-Myc from indirect effects of cell-cycle progression. c-myc null cells can be cultured in standard growth factor-containing medium, yet they have a pronounced cell-cycle defect due solely to the c-myc knockout. Because only the cad gene is reduced in expression in log-phase c-myc null cells, it is clear that none of the other genes that we have
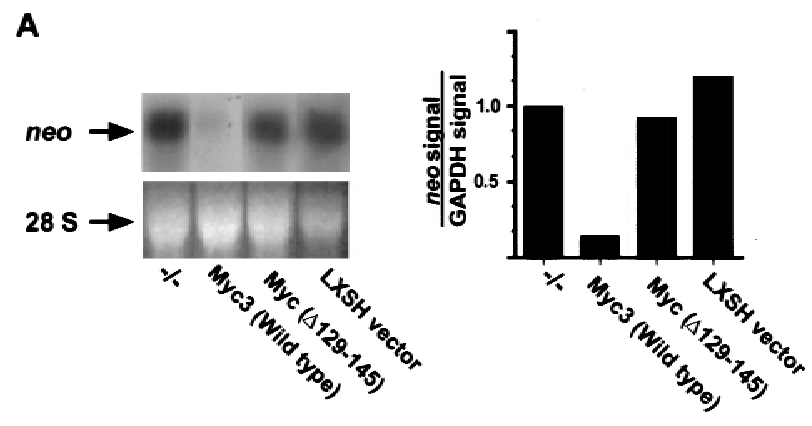

B

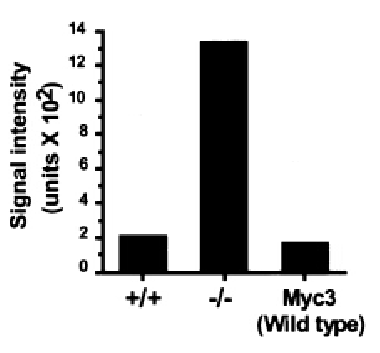

C

Figure 5. c-myc and gadd45 repression in c-myc null cells. $(A)$ (Left) c-myc promoter activity was monitored by Northern blot using a neo probe in c-myc null cells (-/-) or in c-myc null cells reconstituted with wild-type c-Myc, Myc( $\Delta 129-145)$ or vector only. (Right) The neo hybridization signal was normalized to that of GAPDH using a PhosphorImager after reprobing the same Northern blot. (B) Levels of gadd45 mRNA are elevated during log-phase growth in c-myc null cells. Total RNA was analyzed by Northern blot and the level of gadd45 was normalized to GAPDH using a PhosphorImager. (C) RNase protection assay showing levels of gadd45 mRNA during serum stimulation of quiescent cells. There is a 7-fold difference in gadd45 levels between wild-type (solid bars) and c-myc null cells (shaded bars) at $4 \mathrm{hr}$ and a 4.6 -fold difference at $8 \mathrm{hr}$. 
studied are actually transactivated by c-Myc under these growth conditions. We conclude that they are not responsible for the dramatic slow growth phenotype.

Binding sites for Myc/Max heterodimers are expected to occur frequently in the genome and to be recognized by other widely expressed transcription factors such as upstream stimulating factor (USF) and Mnt/Max (Sirito et al. 1994; Hurlin et al. 1997). The CACGTG regulatory element in the cad promoter was cross-linked more readily to USF and Max than to Myc (Boyd and Farnham 1997), indicating that a promoter that shows a substantial Myc dependence is likely to interact with multiple related factors. In addition, transcriptional transactivation can arise from the recruitment of even fortuitous acidic domains to binding sites near a basal promoter (Ma and Ptashne 1987). In transient assays or on induction of an abundantly expressed Myc-ER fusion protein, endogenous factors may be overwhelmed by nonphysiological levels of c-Myc. Thus, the response of a promoter to ectopic c-Myc expression may not be a reliable indicator of in vivo transcriptional regulation.

During the $\mathrm{G}_{0} \rightarrow \mathrm{G}_{1}$ transition, the profile of cdc25A, $M r D b$, and $O D C$ induction has a minor (1.5- to 2-fold) reduction in the early peak level in c-myc null cells, but both wild-type and c-myc null cells reach equivalent levels by $24 \mathrm{hr}$. One of the difficulties in concluding that a particular gene is under the control of c-Myc during serum stimulation has been the inability to differentiate a direct effect of c-Myc on the expression of the gene from an indirect effect resulting from cell-cycle progression. Because c-myc null cells reach S phase more slowly than wild-type cells (Mateyak et al. 1997), the slower induction of $c d c 25 A, M r D b$, and $O D C$ may simply reflect this delayed progression. An alternative possibility is that cMyc contributes to the expression of these genes solely during the transition from quiescence to growth, but the effect of deleting c-myc is similar in magnitude to the effect on genes, such as GAPDH and $L 32$, which have no previous history as targets of c-Myc.

It is interesting to note that the time course of cad induction does not directly parallel c-Myc protein expression. c-Myc protein levels peak at $3 \mathrm{hr}$ after serum stimulation, $\sim 9 \mathrm{hr}$ before peak cad expression. However, after the initial high-level induction, c-Myc continues to be synthesized and levels remain high during the time of peak cad induction (Mateyak et al. 1997), so it is possible that the transcriptional activity of c-Myc may be differentially regulated at distinct points in $\mathrm{G}_{1}$ (Lutterbach and Hann 1994; Colman and Ostrowski 1996). Alteration of the c-Myc/Max binding site in the first exon of cad has shown that this cis element is necessary for induction of a cad reporter construct (Miltenberger et al. 1995), but because other ubiquitous cellular factors can bind to CACGTG, it remains uncertain whether it is c-Myc or some other factor that utilizes this site. However, when combined with in vivo cross-linking (Boyd and Farnham 1997) and the lack of cad induction in cmyc null cells, the data suggest that c-Myc controls cad expression through a direct interaction with the cad gene. Thus the cad promoter should prove informative as a unique transactivation target of c-Myc.

cad encodes a large multisubunit protein that catalyzes the first three steps of pyrimidine biosynthesis (Padgett et al. 1979). An important question is whether reduced cad expression might account for the slow growth phenotype of c-myc null cells. We tested this by culturing c-myc null cells in media supplemented with thymidine and uridine to restore endogenous pyrimidine pools, but there was no rescue of the growth defect (data not shown). Furthermore, ectopic cad expression does not cooperate with a mutated H-ras oncogene in the transformation of primary fibroblasts, suggesting that overexpression of cad is not oncogenic (data not shown). It remains possible that reduced cad expression is one component of the growth defect and that this reduction provides an additive effect in concert with other critical targets that remain to be discovered.

Among genes with known links to cell proliferation, the largest alteration in gene expression in log-phase cells resulting from c-myc knockout is the failure to repress gadd45 mRNA levels. It had been shown previously that overexpression of $\mathrm{v}$-myc represses gadd45 mRNA during log-phase growth and that a Myc-ER fusion protein reduces gadd45 expression when activated with tamoxifen during serum starvation (Marhin et al. 1997). Our results indicate that endogenous levels of cMyc are necessary, as well as sufficient, for the proper control of gadd45 mRNA levels. Ectopic expression of gadd45 has been shown to reduce colony formation in various human tumor lines and NIH-3T3 cells (Zhan et al. 1994; Vairapandi et al. 1996); therefore, the loss of gadd45 suppression may contribute to the slower growth of c-myc null cells. For c-Myc to facilitate exit from $\mathrm{G}_{0}$, it is logical that it should repress gadd45, an inhibitor of cell-cycle progression abundantly expressed at quiescence. Further work will be required to determine the contribution of gadd45 to the overall c-myc null phenotype. The importance of repression in c-Myc function is underscored by an analysis of c-Myc protein forms that retain the ability to repress gene expression but are deficient for transactivation (Xiao et al. 1998).

Enormous effort has been expended in the attempt to define c-Myc target genes by overexpression methods. The loss-of-function approach presented in this study provides a new means to assess potential interactions in the c-myc signaling pathway. The identification of genes in addition to gadd45 and cad that are affected by c-myc knockout should help clarify its role in proliferation and, ultimately, in tumorigenesis.

\section{Materials and methods}

TGR-1 (c-myc wild type) and Ho15.19 (c-myc null) cells were cultured as described (Mateyak et al. 1997). For expression in asynchronously growing cells, RNA was harvested $22-28 \mathrm{hr}$ after plating. For serum stimulation experiments, cells were cultured in $0.1 \%$ serum for $48 \mathrm{hr}$ and the medium was replaced with $10 \%$ serum medium. c-myc rescue cells (HOmyc3) were created by infecting Hol5.19 cells (about passage 30) with the LXSH retrovirus (Miller et al. 1993) containing a full-length mouse c-myc cDNA or mouse c-myc with a deletion in the MbII region (amino acids 129-145) (Brough et al. 1995). The c-Myc protein level in the reconsti- 
tuted lines is two- to fourfold higher than in log-phase c-myc wild-type cells (data not shown).

Total RNA was isolated using a modification of the guanidium-thiocyanate method (Trizol, GIBCO-BRL). RNA was separated on agarose gels containing formaldehyde and transferred to Nytran membranes. All probes were made by random primer labeling of gel-purified cDNA fragments. Blots were washed at a minimum stringency of $0.1 \times$ SSC, $0.1 \%$ SDS at $50^{\circ} \mathrm{C}$. Quantitation of bands was performed using a Molecular Dynamics PhosphorImager. Bands were delimited at high magnification using the rectangle function, and object average was used for background correction. RNase protection assays were performed by standard methods. The RNase protection data in Figure 3, C and D, is normalized to a GAPDH protection probe included in each sample, and the data are plotted according to RNA isolated from an equal cell number.

\section{Acknowledgments}

We thank Maureen Murphy and Arnold Levine for critical reading of the manuscript. We are grateful to our many colleagues for providing $\mathrm{cD}$ NAs. This work was supported by grants from the New Jersey Commission on Cancer Research and the National Institutes of Health to M.D.C. and J.S.

The publication costs of this article were defrayed in part by payment of page charges. This article must therefore be hereby marked 'advertisement' in accordance with 18 USC section 1734 solely to indicate this fact.

\section{References}

Armelin, H., M. Armelin, K. Kelly, T. Stewart, P. Leder, B. Cochran, and C. Stiles. 1984. Functional role for c-myc in mitogenic response to platelet-derived growth factor. Nature 310: 655-660.

Bello-Fernandez, C., G. Packham, and J.L. Cleveland. 1993. The ornithine decarboxylase gene is a transcriptional target of c-Myc. Proc. Natl. Acad. Sci. 90: 7804-7808.

Benvenisty, N., A. Leder, A. Kuo, and P. Leder. 1992. An embryonically expressed gene is a target for c-Myc regulation via the c-Myc-binding sequence. Genes \& Dev. 6: 2513-2523.

Boyd, K.E. and P.J. Farnham. 1997. Myc versus USF: Discrimination at the cad gene is determined by core promoter elements. Mol. Cell. Biol. 17: 2529-2537.

Brough, D.E., T.J. Hofmann, K.B. Ellwood, R.A. Townley, and M.D. Cole. 1995. An essential domain of the c-Myc protein interacts with a nuclear factor that is also required for E1A-mediated transformation. Mol. Cell. Biol. 15: 1536-1544.

Colman, M.S. and M.C. Ostrowski. 1996. The transactivation potential of a c-Myc N-terminal region (residues 92-143) is regulated by growth factor/Ras signaling. Nucleic Acids Res. 24: 1971-1978.

Eilers, M., D. Picard, K.R. Yamamoto, and J.M. Bishop. 1989. Chimeras of myc oncoprotein and steroid receptors cause hormone-dependent transformation of cells. Nature 340: 66-68.

Eilers, M., S. Schirm, and J.M. Bishop. 1991. The MYC protein activates transcription of the $\alpha$-prothymosin gene. EMBO J. 10: 133-142.

Facchini, L.M., S. Chen, W.W. Marhin, J.N. Lear, and L.Z. Penn. 1997. The Myc negative autoregulation mechanism requires Myc-Max association and involves the c-Myc P2 minimal promoter. Mol. Cell. Biol. 17: 100-114.

Galaktionov, K., A.K. Lee, J. Eckstein, G. Draetta, J. Meckler, M. Loda, and D. Beach. 1995. CDC25 phosphatases as potential human oncogenes. Science 269: 1575-1577.

Galaktionov, K., X. Chen, and D. Beach. 1996. CDC25 cell-cycle phosphatase as a target of c-myc. Nature 382: 511-517.

Grandori, C., J. Mac, F. Siebelt, D.E. Ayer, and R.N. Eisenman. 1996 Myc-Max heterodimers activate a DEAD box gene and interact with multiple E box-related sites in vivo. EMBO J. 15: 4344-4357.

Hann, S.R., C.B. Thompson, and R.N. Eisenman. 1985. c-myc oncogene protein synthesis is independent of the cell cycle in human and avian cells. Nature 314: 366-369.

Henriksson, M. and B. Lüscher. 1996. Proteins of the Myc network: Essential regulators of cell growth and differentiation. Adv. Cancer Res 68: 109-182

Hurlin, P., C. Queva, and R. Eisenman. 1997. Mnt, a novel Max-interacting protein is coexpressed with Myc in proliferating cells and me- diates repression at Myc binding sites. Genes \& Dev. 11: 44-58

Jones, R.M., J. Branda, K.A. Johnston, M. Polymenis, M. Gadd, A. Rustige, L. Callanan, and E.V. Schmidt. 1996. An essential E box in the promoter of the gene encoding the mRNA cap binding protein (eukaryotic initiation factor $4 \mathrm{E}$ ) is a target for activation by c-myc. Mol. Cell. Biol. 16: 4754-4764.

Kollmar, R., K. Sukow, S. Sponagle, and P.J. Farnham. 1994. Start site selection at the TATA-less carbamoyl-phosphate synthase (glutamine hydrolyzing//aspartate carbamoyltransferase/dihydroorotase promoter. J. Biol. Chem. 269: 2252-2257.

Kretzner, L., E.M. Blackwood, and R.N. Eisenman. 1992. Myc and Max proteins possess distinct transcriptional activities. Nature 359: 426429.

Lewis, B., H. Shim, Q. Li, C.S. Wu, L.A. Lee, A. Maity, and C.V. Dang. 1997. Identification of putative c-Myc-responsive genes: Characterization of $r c l$, a novel growth-related gene. Mol. Cell. Biol. 17: 4967-4978.

Lutterbach, B. and S.R. Hann. 1994. Hierarchical phosphorylation at Nterminal transformation-sensitive sites in c-Myc protein is regulated by mitogens and in mitosis. Mol. Cell. Biol. 14: 5510-5522.

Ma, J. and M. Ptashne. 1987. A new class of yeast transcriptional activators. Cell 51: 113-119.

Marcu, K.B., S.A. Bossone, and A.J. Patel. 1992. myc function and regulation. Annu. Rev. Biochem. 61: 809-860.

Marhin, W., S. Chen, L. Facchini, A. Fornace, and L.Z. Penn. 1997. Myc represses the growth arrest gene gadd45. Oncogene 14: 2825-2834.

Mateyak, M.K., A.J. Obaya, S. Adachi, and J.M. Sedivy. 1997. Phenotypes of c-Myc-deficient rat fibroblasts isolated by targeted homologous recombination. Cell Growth Differ. 8: 1039-1048.

Miller, A., D. Miller, J. Garcia, and C. Lynch. 1993. Use of retroviral vectors for gene transfer and expression. Methods Enzymol. 217: 581599.

Miltenberger, R.J., K.A. Sukow, and P.J. Farnham. 1995. An E-Box-mediated increase in cad transcription at the $\mathrm{G}_{1} / \mathrm{S}$-phase boundary is suppressed by inhibitory c-Myc mutants. Mol. Cell. Biol. 15: 2527-2535.

Padgett, R.A., G.M. Wahl, P.F. Coleman, and G.R. Stark. 1979. N-(Phosphonacetyl)-L-aspartate-resistant hamster cells overaccumulate a single mRNA coding for the multifunctional protein that catalyzes the first steps of UMP synthesis. J. Biol. Chem. 254: 974-980.

Penn, L.J.Z., M.W. Brooks, E.M. Laufer, T.D. Littlewood, J.P. Morgenstern, G.I. Evan, W.M.F. Lee, and H. Land. 1990. Domains of human c-Myc protein required for autosuppression and cooperation with ras oncogenes are overlapping. Mol. Cell. Biol. 10: 4961-4966.

Reisman, D., N.B. Elkind, B. Roy, J. Beamon, and V. Rotter. 1993. c-Myc trans-activates the $\mathrm{p} 53$ promoter through a required downstream CACGTG motif. Cell Growth Differ. 4: 57-65.

Shim, H., C. Dolde, B. Lewis, C.S. Wu, G. Dang, R.A. Jungmann, R. Dalla-Favera, and C.V. Dang. 1997. c-Myc transactivation of LDH-A Implications for tumor metabolism and growth. Proc. Nat1. Acad. Sci. 94: 6658-6663.

Sirito, M., Q. Lin, T. Maity, and M. Sawadogo. 1994. Ubiquitous expression of the 43- and 44-kDa forms of transcription factor USF in mammalian cells. Nucleic Acids Res. 22: 427-433.

Tsuneoka, M., F. Nakano, H. Ohgusu, and E. Mekada. 1997. c-myc activates RCCl gene expression through E-box elements. Oncogene 14: $2301-2311$.

Vairapandi, M., A.G. Balliet, A.J. Fornace, B. Hoffman, and D.A. Lieberman. 1996. The differentiation primary response gene Myd118, related to GADD45, encodes for a nuclear protein which interacts with PCNA and $\mathrm{p} 21^{\text {Waf1/Cip1 }}$. Oncogene 12: 2579-2594.

Wagner, A.J., C. Meyers, L. Laimins, and N. Hay. 1993. c-Myc induces the expression and activity of ornithine decarboxylase. Cell Growth Differ. 4: 879-883.

Xiao, Q. G.Claassen, J. Shi, S. Adachi, J. Sedivy, and S.R. Hann. 1998. Transactivation-defective c-MycS retains the ability to regulate proliferation and apoptosis. Genes \& Dev. 12: (this issue).

Yokoyama, K., and F. Imamoto. 1987. Transcriptional control of the endogenous MYC protooncogene by antisense RNA. Proc. Natl. Acad. Sci. 84: 7363-7367.

Zhan, Q., K.A. Lord, I. Alamo, M.C. Hollander, F. Carrier, D. Ron, K. Kohn, B. Hoffman, L.D.A., and A.J. Fornace. 1994. The gadd and Myd genes define a novel set of mammalian genes encoding acidic proteins that synergestically suppress cell growth. Mol. Cell Biol. 14: 23612371. 


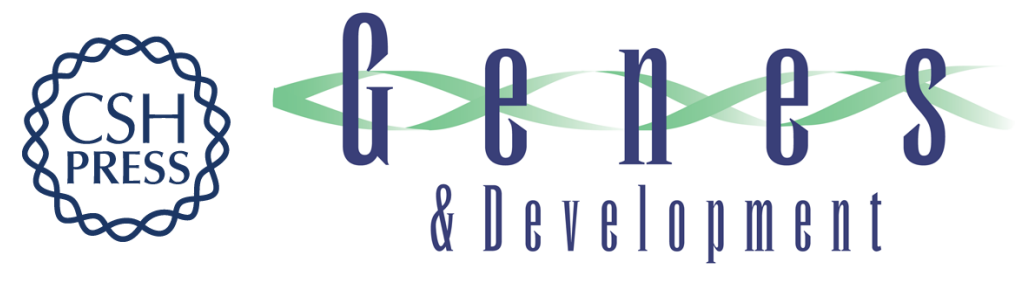

\section{c-myc null cells misregulate cad and gadd45 but not other proposed c-Myc targets}

Andrew Bush, Maria Mateyak, Kerri Dugan, et al.

Genes Dev. 1998, 12:

Access the most recent version at doi:10.1101/gad.12.24.3797

References

This article cites 36 articles, 20 of which can be accessed free at: http://genesdev.cshlp.org/content/12/24/3797.full.html\#ref-list-1

\section{License}

Email Alerting

Receive free email alerts when new articles cite this article - sign up in the box at the top Service right corner of the article or click here.

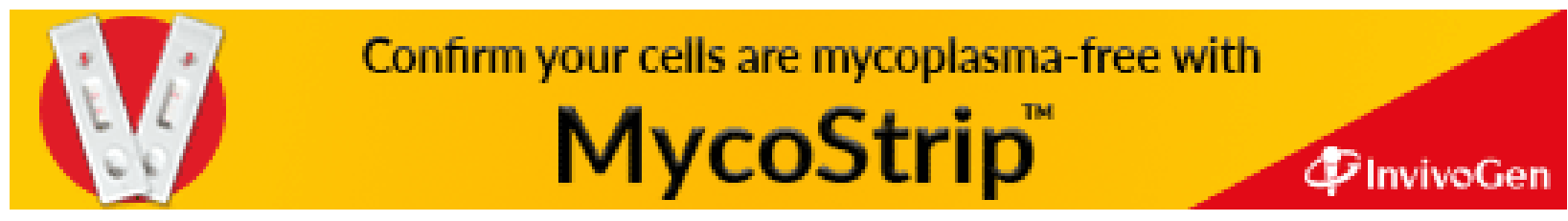

